\title{
Correction to: Shallowness of tropical low clouds as a predictor of climate models' response to warming
}

\author{
Florent Brient ${ }^{1} \cdot$ Tapio Schneider $^{1,2} \cdot$ Zhihong Tan $^{1,2} \cdot$ Sandrine Bony ${ }^{3} \cdot$ Xin $\mathrm{Qu}^{4} \cdot$ Alex Hall $^{4}$
}

Published online: 3 February 2021

c) Springer-Verlag GmbH Germany, part of Springer Nature 2021

\section{Correction to: Clim Dyn (2016) 47:433-449 https://doi.org/10.1007/s00382-015-2846-0}

Several mistakes occur in the original article that need to be notified.

In Table 1, the shallowness index $(\gamma)$ for the HadGEM2ES model should be $44 \pm 3$, instead of $4 \pm 3$.

In Fig. $4 a$, the $x$-axis should start with the value 0 instead of -20 .

The convective drying index $\left(\epsilon_{c}\right)$ is a dimensionless quantity (ratio). How-ever, an percent unit (\%) was wrongly associated with $\epsilon_{\mathrm{c}}$ in Figure 7 and Table 3.

Finally, the signs of $\epsilon_{c}$ in Table 3 are wrong. However, their values in Figure 7 remain exact. The updated table is available on page 2 .

These corrections do not change the conclusions of the paper.

\begin{tabular}{lllllllll}
\hline Model acronym & $\gamma(\%)$ & $\in_{\mathrm{c}}(-)$ & $\begin{array}{l}K_{m p}\left(\mathrm{~m}^{2}\right. \\
\left.\mathrm{s}^{-1}\right)\end{array}$ & \multicolumn{4}{l}{$\begin{array}{l}\text { SHF, LHF, B } \\
\left(\mathrm{W} \mathrm{m} \mathrm{m}^{-2}\right)\end{array}$} \\
\hline MIROC5 & 4 & 42 & 0.6 & 8.4 & 4.1 & 150 & 14.6 \\
& & 2.0 & 0.00 & -0.10 & -0.18 & 5.6 & 0.21 \\
MRI- & 11 & 34 & 1.0 & 8.5 & 11.8 & 145 & 22.0 \\
CGCM3 & & & & & & & \\
& & -0.6 & -0.02 & 0.20 & -0.49 & 6.3 & -0.05
\end{tabular}

The original article can be found online at https://doi.org/10.1007/ s00382-015-2846-0.

\section{Florent Brient}

florent.brient@erdw.ethz.ch

1 Department of Earth Sciences, ETH Zurich, Zurich, Switzerland

2 California Institute of Technology, Pasadena, CA, USA

3 Laboratoire de Météorologie Dynamique (LMD/IPSL), Université Pierre et Marie Curie, CNRS, Paris, France

4 Department of Atmospheric and Oceanic Sciences, University of California, PO Box 951565, Los Angeles, CA 90095-1565, USA

\begin{tabular}{|c|c|c|c|c|c|c|c|}
\hline \multicolumn{2}{|c|}{ Model acronym } & \multirow{2}{*}{$\begin{array}{l}\gamma(\%) \\
70\end{array}$} & \multirow{2}{*}{$\frac{\epsilon_{\mathrm{c}}(-)}{2.1}$} & \multirow{2}{*}{$\begin{array}{l}\begin{array}{l}K_{m p}\left(\mathrm{~m}^{2}\right. \\
\left.\mathrm{s}^{-1}\right)\end{array} \\
6.4\end{array}$} & \multicolumn{3}{|c|}{$\begin{array}{l}\text { SHF, LHF, B } \\
\left(\mathrm{W} \mathrm{m}^{-2}\right)\end{array}$} \\
\hline MPI-ESM- & 15 & & & & 12.9 & 139 & 22.6 \\
\hline & & -1.9 & -0.05 & 0.16 & -0.50 & 5.9 & -0.10 \\
\hline \multirow{2}{*}{$\begin{array}{l}\text { HadGEM2- } \\
\text { ES }\end{array}$} & 17 & 46 & 0.7 & 8.1 & 10.7 & 150 & 21.2 \\
\hline & & 1.1 & 0.02 & -0.18 & -0.28 & 5.2 & 0.09 \\
\hline \multirow{2}{*}{$\begin{array}{l}\text { IPSL- } \\
\text { CM5A- } \\
\text { LR }\end{array}$} & 21 & 65 & 2.3 & 5.8 & 18.0 & 135 & 27.4 \\
\hline & & -2.8 & 0.00 & 0.28 & -0.57 & 6.4 & -0.12 \\
\hline \multirow{2}{*}{\multicolumn{2}{|c|}{$\begin{array}{r}\text { IPSLA } \\
\text { SCM }\end{array}$}} & 69 & 3.1 & 3.9 & 12.8 & 119 & 21.1 \\
\hline & & -4.2 & 0.40 & 0.10 & -0.64 & 3.7 & -0.38 \\
\hline \multirow{2}{*}{\multicolumn{2}{|c|}{$\begin{array}{l}\text { IPSLA } \\
\text { SCM (no } \\
\text { cv) }\end{array}$}} & 40 & 0.0 & 3.2 & 11.4 & 111 & 19.1 \\
\hline & & 1.1 & 0.00 & -0.02 & -0.17 & 4.0 & 0.11 \\
\hline
\end{tabular}

Table: Corrected Table 3 with updated $\epsilon_{\mathrm{c}}$ values.

Publisher's Note Springer Nature remains neutral with regard to jurisdictional claims in published maps and institutional affiliations. 